\title{
Dispersion relations of charmonia above $T_{c}$
}

\author{
Masakiyo Kitazawa ${ }^{1,2, \star}$, Atsuro Ikeda ${ }^{1}$, and Masayuki Asakawa ${ }^{1}$ \\ ${ }^{1}$ Department of Physics, Osaka University, Osaka, Osaka 560-0043, Japan \\ ${ }^{2} J$-PARC Branch, KEK Theory Center, Institute of Particle and Nuclear Studies, KEK, 203-1, Shirakata, \\ Tokai, Ibaraki 319-1106, Japan
}

\begin{abstract}
We study spectral properties of charmonia in the vector and pseudoscalar channels at nonzero momenta on quenched lattices focusing on the dispersion relation and the weight of the peak. The spectral functions of charmonia are studied by the maximum entropy method with the lattice Euclidean correlation functions on the anisotropic quenched lattices. The errors of the dispersion relations and the residues of the peak are analysed in the maximum entropy method. We find a significant increase of the masses of charmonia in medium. We also find that the functional form of the dispersion relations is not changed from that in the vacuum within the error even at $T=1.6 T_{c}$.
\end{abstract}

Preprint number: J-PARC-TH-0111

\section{Introduction}

Heavy quarkonia are unique observables in relativistic heavy ion collisions. Because of their heavy mass, heavy quarkonia act as impurities in the hot medium created in heavy ion collisions. Therefore, understanding their properties in hot medium near and above the critical temperature $T_{\mathrm{c}}$ of the deconfinement phase transition is an important subject. For example, by understanding the stabilities of heavy quarkonia, their yields in heavy ion collisions would be used as a signal of the formation of the quark-gluon plasma [1]. The study of the properties of charmonia at finite temperature in lattice QCD numerical simulations is one of the longstanding subjects on the lattice [2-8]. In the lattice simulations which rely on the imaginary time formalism, however, one cannot analyze dynamical properties encoded in spectral functions directly. Instead, only Euclidean correlation functions are calculable on the lattice. The maximum entropy method (MEM) is a useful method to perform this analytic continuation on the basis of probability theory [9]. The studies of charmonium spectral functions with MEM qualitatively agree with each other in that the charmonia in the vector and pseudoscalar channels survive up to around $T \simeq 1.5 T_{\mathrm{c}}[2,3,8,10]$.

In the previous studies on heavy quarkonia on the lattice, the analyses have been performed only for zero momentum with a few exceptions. The spectral function with zero momentum represents the spectral properties of a charmonium at rest in medium. On the other hand, charmonia in the hot medium created by heavy ion collisions typically have nonnegligible velocity against the rest frame

${ }^{\star}$ Speaker, e-mail: kitazawa@phys.sci.osaka-u.ac.jp 
of the medium because charmonia generated by hard processes in the early stage can have large momentum. The finiteness of the velocity of charmonia may modify their properties, such as the stability and the dispersion relation, i.e. the momentum dependence of energy. It thus is interesting to explore the momentum dependence of spectral functions of charmonia and their dispersion relations near and above $T_{\mathrm{c}}$ on the lattice.

In this study we explore the properties of charmonia in the vector and pseudoscalar channels, corresponding to $J / \psi$ and $\eta_{c}$, respectively, at nonzero momenta on anisotropic quenched lattices [11]. In addition to the standard analysis of the spectral functions in MEM, we study the dispersion relations and the momentum dependence of the spectral weights of the $J / \psi$ and $\eta_{c}$ peaks on the basis of MEM. To perform the measurement of the dispersion relation with a quantitative error analysis in MEM, we analyze the center of weight of the peak in the spectral function. As we will see later, this quantity is identical to the peak position for sufficiently narrow peaks, but error analysis can be carried out in MEM. Similarly, we analyze the weight of the peak, which corresponds to the residue of the peak, with the error analysis. We find that the masses of $J / \psi$ and $\eta_{c}$ defined by the dispersion relation at zero momentum show significant increase as temperature $(T)$ is raised. It is also found that the dispersion relation of charmonia continues to take the Lorentz covariant form, i.e. the same form as in the vacuum, even well above $T_{\mathrm{c}}$ within the error.

\section{Euclidean correlator and spectral function}

Dynamical properties of charmonia are encoded in the Euclidean correlators $G^{\operatorname{lm}}(\tau, \vec{p})=$ $\int d^{3} x e^{i \vec{p} \cdot \vec{x}}\left\langle J^{l}(\tau, \vec{x}) J^{m_{\dagger}^{\dagger}}(0, \overrightarrow{0})\right\rangle$, where the imaginary time $\tau$ is restricted to the interval $0 \leq \tau<1 / T$ and $J^{l}(\tau, \vec{x})=\bar{c}(\tau, \vec{x}) i \gamma^{l} c(\tau, \vec{x})$ is the local interpolating operator in the Heisenberg representation with the charm quark field $c(\tau, \vec{x})$ with $l=0,1,2$, and 3 for the vector channel and $l=5$ for the pseudoscalar channel. The spectral function is defined as $A^{l m}(\omega, \vec{p})=(1 / \pi) \operatorname{Im}\left[G_{\mathrm{R}}^{l m}(\omega, \vec{p})\right]$, where

$$
G_{\mathrm{R}}^{l m}(\omega, \vec{p})=\int_{-\infty}^{\infty} d t \int d^{3} \vec{x} e^{-i \vec{p} \cdot \vec{x}+i \omega t} G_{\mathrm{R}}^{l m}(t, \vec{x}), \quad G_{\mathrm{R}}^{l m}(t, \vec{x})=i \theta(t)\left\langle\left[J^{l}(t, \vec{x}), J^{m \dagger}(0, \overrightarrow{0})\right]\right\rangle .
$$

The diagonal components of the spectral functions $A^{l l}(\omega, \vec{p})$ are related to $G^{l m}(\tau, \vec{p})$ by the Laplace-like transformation as

$$
G^{l l}(\tau, \vec{p})=\int_{0}^{\infty} K(\tau, \omega) A^{l l}(\omega, \vec{p}) d \omega,
$$

with $K(\tau, \omega)=\left(e^{-\tau \omega}+e^{-(1 / T-\tau) \omega}\right) /\left(1-e^{-\omega / T}\right)$. In the following, we represent the diagonal components of the spectral functions as $A^{l}(\omega, \vec{p})=A^{l l}(\omega, \vec{p})$.

When there is a bound state which couples to $J^{l}$, the corresponding spectral function has a delta function $Z \delta\left(\omega^{2}-E(p)^{2}\right)=(Z / 2 E(p)) \delta(\omega-E(p))$, where $E(p)$ is the dispersion relation of the bound state with $p=|\vec{p}|$ and $Z>0$ is the residue. Because of Lorentz invariance, $E(p)$ in the vacuum is given by

$$
E(p)=\sqrt{m^{2}+p^{2}}
$$

where $m$ is the mass of the bound state. At nonzero temperature, the delta function is replaced by a smooth function with a peak. Because Lorentz invariance is lost in medium, $Z$ can depend on momentum. The dispersion relation $E(p)$ can also be modified from Eq. (3).

At finite temperature, $A^{\mu v}(\omega, \vec{p})$ is decomposed into the transverse and longitudinal components as

$$
A^{\mu v}(\omega, \vec{p})=P_{\mathrm{T}}^{\mu v} A_{\mathrm{T}}(\omega, p)+P_{\mathrm{L}}^{\mu v} A_{\mathrm{L}}(\omega, p),
$$


with the projection operators onto the transverse and longitudinal components, $P_{\mathrm{T}}$ and $P_{\mathrm{L}}$. The transverse and longitudinal spectral functions $A_{\mathrm{T}}(\omega, p)$ and $A_{\mathrm{L}}(\omega, p)$ are identical in the vacuum, $A_{\mathrm{T}}(\omega, p)=A_{\mathrm{L}}(\omega, p)=A_{\mathrm{V}}(\omega, p)$. When the momentum is taken as $\vec{p}=(p, 0,0), A_{\mathrm{T}}(\omega, p)$ and $A_{\mathrm{L}}(\omega, p)$ are related to $A^{i}(\omega, \vec{p})$ as

$$
A_{\mathrm{T}}(\omega, p)=\frac{1}{2}\left(A^{2}(\omega, \vec{p})+A^{3}(\omega, \vec{p})\right), \quad A_{\mathrm{L}}(\omega, p)=\frac{\omega^{2}-p^{2}}{\omega^{2}} A^{1}(\omega, \vec{p}) .
$$

From the general property of the spectral function, $A^{l}(\omega, \vec{p})$ are semi-positive for $\omega>0$. On the other hand, Eq. (5) shows that $A_{\mathrm{L}}(\omega, p)$ is semi-negative in the space-like region $0<\omega<p$.

\section{Maximum entropy method and dispersion relation}

To obtain a spectral function from a lattice Euclidean correlator, we have to take the inverse transformation of Eq. (2). MEM [9, 12] is a method to infer the most probable image of the spectral function from a limited number of data points for a Euclidean correlator on the basis of Bayes' theorem. In this method, the spectral function is determined by the probability $P(A, \alpha)=\exp \left[\alpha S(A)-\frac{1}{2} \chi^{2}(A)\right]$, with chi-square $\chi^{2}(A)$ and the Shannon-Jaynes entropy $S=\int_{0}^{\infty}[A(\omega)-m(\omega)-A(\omega) \log (A(\omega) / m(\omega))] d \omega$. The default model $m(\omega)$ expresses prior knowledge. The parameter $\alpha$ controls the relative weight between $\chi^{2}$ and $S$. The final output image $A_{\text {out }}(\omega)$ is obtained by integrating $A(\omega)$ with a weight $P(A, \alpha)$ over $\alpha$ and $A$ space as $A_{\text {out }}(\omega)=\langle A(\omega)\rangle$, where

$$
\langle O\rangle=\frac{1}{Z_{\mathrm{P}}} \int d \alpha \int[d A] P(A, \alpha) O,
$$

is the average over the plausibility $P(A, \alpha)$ with $Z_{\mathrm{P}} \equiv \int d \alpha \int[d A] P(A, \alpha)$. Here, the measure is defined as $[d A] \equiv \lim _{N_{\omega} \rightarrow \infty} \prod_{l=1}^{N_{\omega}}\left(d A_{l} / \sqrt{A_{l}}\right)$, where the discretized spectral function $A_{l}=A\left(\omega_{l}\right)$ with discrete $\omega$ values $\omega_{l}$ [9]. When $P(A, \alpha)$ is sharply peaked around $A_{\alpha}(\omega), A_{\text {out }}(\omega)$ is well approximated as $A_{\text {out }}(\omega) \simeq \frac{1}{Z_{\mathrm{P}}} \int d \alpha A_{\alpha}(\omega) P(\alpha)$, with $P(\alpha) \equiv \int[d A] P(A, \alpha)$.

A characteristic of MEM is that this method enables us to estimate the error of quantities given by the integral of a function of $A_{\text {out }}(\omega)$ quantitatively. Let us consider a quantity given by the weighted integral of $A(\omega)$ with a weight function $f(\omega)$ and an interval $I=\left[\omega_{\min }, \omega_{\max }\right], W=\int_{I} f(\omega) A(\omega) d \omega$. In MEM, the average and the error of $W$ is estimated as

$$
\langle W\rangle=\left\langle\left\langle\int_{I} d \omega f(\omega) A(\omega)\right\rangle, \quad \Delta W=\sqrt{\left\langle(\delta W)^{2}\right\rangle},\right.
$$

respectively, where $\delta W=W-\langle W\rangle$.

Typically, the magnitude of the error estimated in this way becomes larger as the interval $I$ becomes narrower. In particular, when one makes the error estimate of $A_{\text {out }}(\omega)$ at a given $\omega$ with $f\left(\omega^{\prime}\right)=\delta\left(\omega^{\prime}-\omega\right)$, one obtains a huge error $\Delta A_{\text {out }}(\omega) \gg A_{\text {out }}(\omega)$. This means that the functional form of $A_{\text {out }}(\omega)$ itself does not have quantitative meaning. The values of the position and width of the peak do not have statistically relevant meanings, either. In order to obtain a moderate value of the error, the interval $I$ has to be chosen sufficiently large.

In this study, we focus on the dispersion relation and the momentum dependence of the spectral weight of the $J / \psi$ and $\eta_{c}$. To study these quantities with error estimates in MEM, we consider quantities

$$
\bar{Z}(p)=\int_{I} d \omega 2 \omega A(\omega, p), \quad \bar{E}(p)=\frac{\int_{I} d \omega \omega\left(A(\omega, p) / \omega^{2}\right)}{\int_{I} d \omega\left(A(\omega, p) / \omega^{2}\right)},
$$


for a peak in a spectral function $A(\omega, p)$, where $I$ is the interval of $\omega$ which covers the peak structure. One easily finds that for the delta function we have $\bar{Z}(p)=Z$ and $\bar{E}(p)=E(p)$. Note that $\bar{Z}(p)$ and $\bar{E}(p)$ are meaningful only for well isolated peaks for which such a choice of $I$ is possible. Since $\bar{Z}(p)$ and $\bar{E}(p)$ are given by the integral of $A(\omega)$ as in Eq. (8), these are quantities which can be estimated in MEM with error.

\section{Simulation set up}

In this study [11], we measure the momentum dependence of charmonium correlation functions in the vector and pseudoscalar channels on quenched anisotropic lattices with the standard Wilson gauge action and Wilson fermion. The simulation parameters are $\beta=7.0$, the bare anisotropy $\xi_{0}=3.5$, the spatial hopping parameter $\kappa_{\sigma}=0.08285$, and the fermion anisotropy $\gamma_{F}=3.476$ [2]. The anisotropy $\xi=a_{\sigma} / a_{\tau}$ is 4 with the lattice spacings along spatial and temporal directions, $a_{\sigma}$ and $a_{\tau}$, respectively. We use lattices of volumes $64^{3} \times N_{\tau}$ with $44 \leq N_{\tau} \leq 96$ corresponding to $0.78 T_{c}<T<1.70 T_{c}$. Our lattice has relatively large spatial volume $L_{\sigma} \simeq 2.5 \mathrm{fm}$. The large spatial extent enables a detailed study of the momentum dependence of the quantities on the lattice. With a periodic boundary condition along spatial direction, the momentum of bosons on the lattice is discretized as $p_{i}=\left(2 / a_{\sigma}\right) \sin \left(\pi \hat{p}_{i} / N_{\sigma}\right)$. In the analysis of Euclidean correlators we take the momentum along 1 direction, i.e. $\vec{p}=(p, 0,0)$. The largest lattice with $N_{\tau}=96$ and $T / T_{\mathrm{c}}=0.78$ is regarded as the vacuum one, in which the medium effects are well suppressed. The error to signal ratios of the correlators around the mid point $\tau=1 /(2 T)$ at $p=0$ are about $0.1 \sim 0.2 \%$.

In the MEM analysis of spectral functions, we use a default model $m(\omega)=m_{0} \omega^{2}+m_{1} T \omega[13]$, where $m_{0}=1.15$ for the pseudoscalar channel and $m_{0}=0.40$ for the vector channel [2]. We analyzed the default model dependence of the reconstructed spectral function by changing $m_{0}$ and $m_{1}: m_{0}$ is varied in the 50\% range from the above values, while the dependence on $m_{1}$ is also checked in the range $0 \leq m_{1} \leq 2.0$. We found that the dependence on $m_{0}$ and $m_{1}$ is well suppressed in this range near the peak of the $J / \psi$ and $\eta_{c}$; the change of the spectral image caused by the variation of $m_{0}$ and $m_{1}$ in these ranges around the charmonium peak is less than a few percent. In the following analyses we thus set $m_{1}=0$. For nonzero $p$, we have also performed MEM analysis with a default model $m(\omega)=\max \left(m_{0}\left(\omega^{2}-p^{2}\right), m_{0} \epsilon\right)$ with several choices of small parameter $\epsilon$. We, however, found that the default model dependence of our results is well suppressed again around the peak of the $J / \psi$ and $\eta_{c}$, and the following numerical results hardly change.

For the analysis of $A_{\mathrm{T}}(\omega, p)$ and $A_{\mathrm{PS}}(\omega, p)$, we reconstruct them from the corresponding correlators $G_{\mathrm{T}}(\tau, p)=\left(G^{2}(\tau, \vec{p})+G^{3}(\tau, \vec{p})\right) / 2$ and $G_{\mathrm{PS}}(\tau, p)=G^{5}(\tau, \vec{p})$. On the other hand, $A_{\mathrm{L}}(\omega, p)$ can become negative and one cannot apply MEM to this channel directly. We thus analyze $A^{1}(\omega, \vec{p})$ from $G^{1}(\tau, \vec{p})$ with MEM and obtain $A_{\mathrm{L}}(\omega, p)$ using Eq. (5).

In the analysis of the dispersion relation and the weight of the peak Eq. (8), the interval $I=$ $\left[\omega_{\min }, \omega_{\max }\right]$ has to be chosen appropriately. In this study, we set $\omega_{\min }=3 \mathrm{GeV}$, while for the upper bound $\omega_{\max }$ we use the value of $\omega$ at which the spectral function takes the first local minimum on the right of the peak corresponding to the $J / \psi$ or $\eta_{c}$. We found that our numerical results for $\bar{Z}(p)$ and $\bar{E}(p)$, as well as their errors, are insensitive to the choice of the lower bound $\omega_{\min }$; for example, these quantities do not change within the numerical precision even if $\omega_{\min }$ is set to $2 \mathrm{GeV}$. Our numerical analysis suggests that the results of $\bar{Z}(p)$ and $\bar{E}(p)$ hardly change for a variation of the lower and/or upper limits of $I$ in the range where the reconstructed image takes a small value [11]. 

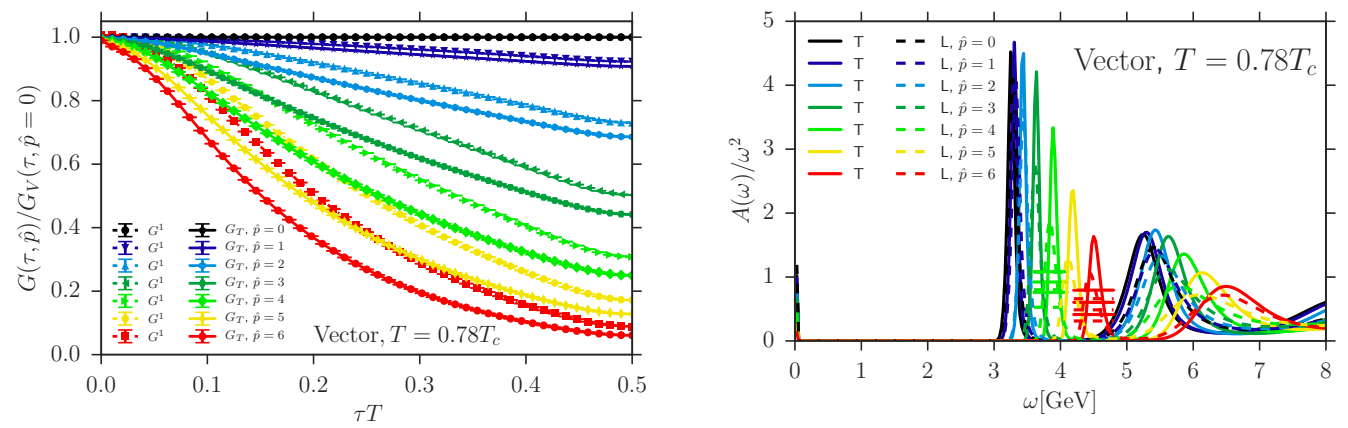

Figure 1. Momentum dependence of correlation functions for the vector channel, $G^{1}(\tau, \vec{p})$ and $G_{\mathrm{T}}(\tau, p)$, normalized by the correlation function with zero momentum $G_{\mathrm{V}}(\tau, 0)$ (left) and the spectral functions $A_{\mathrm{T}}(\omega, p)$ and $A_{\mathrm{L}}(\omega, p)$ in the vector channel at $T=0.78 T_{\mathrm{c}}$ (right) [11].

\section{Numerical results}

\subsection{Correlators and spectral functions}

We first see the momentum dependence of the correlation functions. The left panel of figure 1 shows the correlation functions $G^{1}(\tau, \vec{p})$ and $G_{\mathrm{T}}(\tau, p)$ in the vector channel normalized by those with zero momentum for various values of $\hat{p}$ at $T=0.78 T_{\mathrm{c}}$. In the figure, the ratios $G^{1}(\tau, \vec{p}) / G_{\mathrm{V}}(\tau, 0)$ and $G_{\mathrm{T}}(\tau, p) / G_{\mathrm{V}}(\tau, 0)$ are plotted by the dashed and solid lines, respectively. The figure shows that $G^{1}(\tau, \vec{p})$ and $G_{\mathrm{T}}(\tau, p)$ behave differently. As momentum become larger, the separation becomes more prominent with $G_{\mathrm{T}}(\tau, p)<G^{1}(\tau, \vec{p})$. In the right panel of figure 1 , we show the momentum dependence of the spectral functions in the vector channel, $A_{\mathrm{T}}(\omega, p)$ and $A_{\mathrm{L}}(\omega, p)$, for $T=0.78 T_{\mathrm{c}}$. To see the separation of the transverse and longitudinal channels, we show the errors for the averages of $A_{\mathrm{T}}(\omega, p)$ and $A_{\mathrm{L}}(\omega, p)$ with the same energy interval for $\hat{p}=4$ and 6 . From the figure, one observes that the spectral functions in the transverse and longitudinal channels agree with each other within the error. This result is consistent with the vacuum property of the spectral functions discussed in Sect. 2. It, however, is worth emphasizing that this agreement is obtained although $A_{\mathrm{T}}(\omega, p)$ and $A_{\mathrm{L}}(\omega, p)$ are constructed from completely different correlation functions shown in the right panel of figure 1 .

In figure 2, we show the momentum dependence of the spectral functions in the vector and pseudoscalar channels at $T=1.62 T_{\mathrm{c}}$. One sees that the peaks corresponding to the $J / \psi$ and $\eta_{c}$ survive even at this temperature. Figure 2 also shows that the peaks corresponding to the $J / \psi$ and $\eta_{c}$ are well isolated from the second structure in the spectral functions. This suggests that the dependence of $\bar{Z}(p)$ and $\bar{E}(p)$ on $\omega_{\max }$ is suppressed so that these quantities can be analyzed with small ambiguity.

\subsection{Residue and dispersion relation}

Next, we turn to $\bar{Z}(p)$ and $\bar{E}(p)$. In figure 3, we show the momentum dependence of $\bar{Z}(p)$ obtained with Eq. (8) for the vector and pseudoscalar channels for $T / T_{\mathrm{c}}=0.78,1.49$, and 1.62. In the figure, the normalized results, $\bar{Z}(p) / \bar{Z}(0)$, are plotted in order to see the momentum dependence of $\bar{Z}(p)$. The figure shows that $\bar{Z}(p)$ does not have momentum dependence within the error for all the temperatures and all the channels for which we carried out analysis. This result is reasonable for $T / T_{\mathrm{c}}=0.78$, at which the medium effects should be well suppressed. Our analysis, however, shows that $\bar{Z}(p)$ is insensitive to $p$ even at $T / T_{\mathrm{c}}=1.49$ and 1.62 , which is a nontrivial result. 

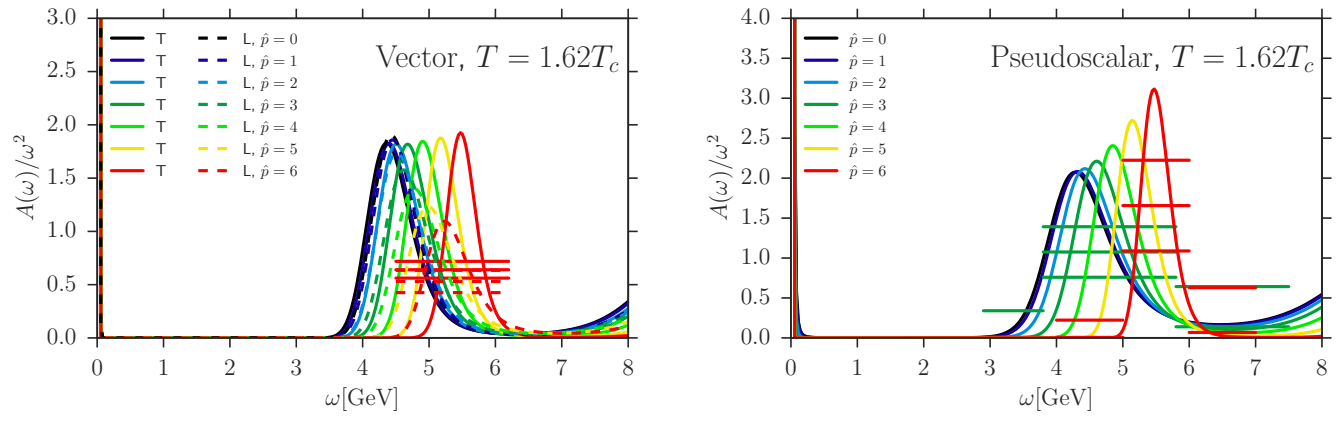

Figure 2. Momentum dependence of the spectral functions at $T=1.62 T_{\mathrm{c}}$. The left and right panels show the vector and pseudoscalar channels, respectively [11]. The error bars are shown for averages of the spectral functions for the vector channel at $\hat{p}=6$ and for the pseudoscalar channel at $\hat{p}=3$ and 6 .
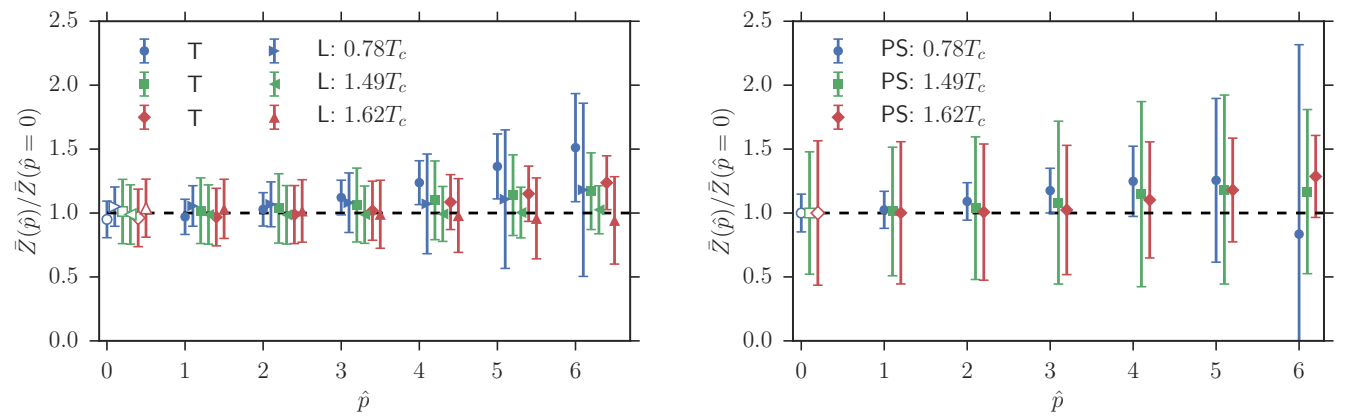

Figure 3. Ratio of the residues of the peaks, $\bar{Z}(p) / \bar{Z}(0)$, corresponding to the $J / \psi$ (left) and $\eta_{c}$ (right) [11]. The transverse $(\mathrm{T})$ and longitudinal $(\mathrm{L})$ components are shown in the left panel.

Table 1. Masses of the ground states of the charmonia in the vector and pseudoscalar channels $\bar{m}=\bar{E}(0)$.

\begin{tabular}{c|ccc}
\hline$T / T_{\mathrm{c}}$ & 0.78 & 1.49 & 1.62 \\
\hline$J / \psi$ & $3.24(6)$ & $4.30(16)$ & $4.47(16)$ \\
$\eta_{c}$ & $3.19(5)$ & $4.24(31)$ & $4.49(48)$ \\
\hline
\end{tabular}

We note that the errors of $\bar{Z}(p) / \bar{Z}(0)$ in figure 3 would be reduced if we take into account the correlation between $\bar{Z}(p)$ and $\bar{Z}(0)$. In order to estimate the correlation, however, one has to perform the MEM analysis for two different correlation functions in a single analysis. Because we perform the MEM analysis for individual momenta, this correlation cannot be estimated in our analysis.

To see the medium effects on the dispersion relation, we show the results on $\bar{E}(p)$ in figure 4 . In the figure, we plot the square of this quantity $(\bar{E}(p))^{2}$ as a function of $p^{2}$, since this plot is convenient to see the deviation of $\bar{E}(p)$ from the vacuum dispersion relation Eq. (3). From the figure, one first observes that the masses of the charmonia, defined by $\bar{m}=\bar{E}(0)$, become larger as $T$ is increased. The values of $\bar{m}$ in the vector and pseudoscalar channels at $T / T_{\mathrm{c}}=0.78,1.49$, and 1.62 are listed 

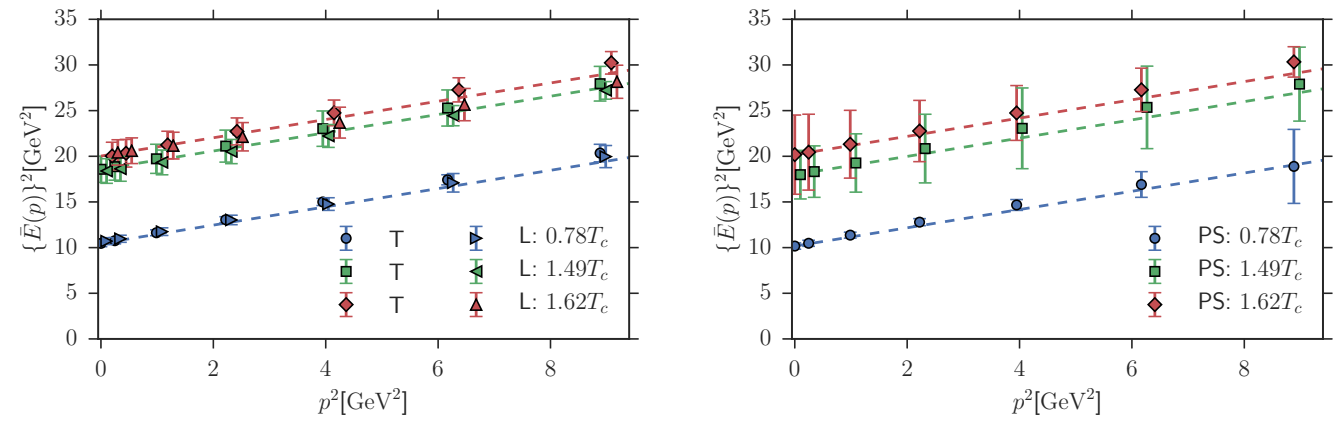

Figure 4. Dispersion relations $\bar{E}(p)$ of the $J / \psi$ (left) and $\eta_{c}$ (right) for $T / T_{\mathrm{c}}=0.78,1.49$, and 1.62 [11]. The dashed lines indicate the vacuum dispersion relation Eq. (3) with $m=\bar{E}(0)$.

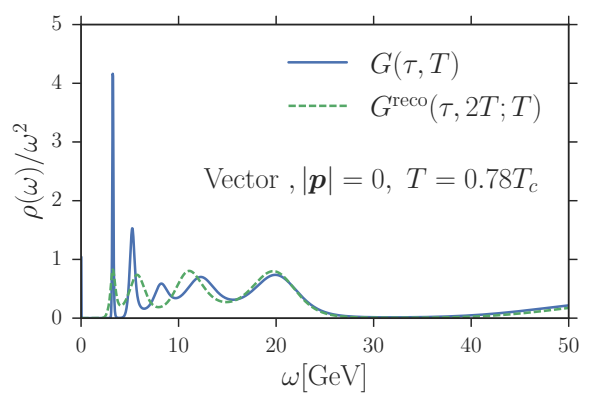

Figure 5. Comparison of spectral functions in vector channel at $T=0.78 T_{c}$ obtained with the conventional and reconstructed correlators $G(\tau)$ and $G^{\text {reco }}(\tau, 2 T ; T)$.

in table 1. Although such a mass shift in MEM analyses were suggested in previous study [14], our analysis confirms the medium effects on the mass of the charmonia with a quantitative error analysis.

In figure 4, the vacuum dispersion relation Eq. (3) with $m=\bar{m}$ is shown by the dashed lines. The figure shows that the functional form of $\bar{E}(p)$ is consistent with Eq. (3) within statistics even at $T / T_{\mathrm{c}}=1.49$ and 1.62 .

\subsection{Check of $N_{\tau}$ dependence}

So far, we studied the $T$ dependence of the dispersion relations by changing $N_{\tau}$. It, however, is known that the quality of MEM analysis is sensitive to the value of $N_{\tau}$. Therefore, it is possible that the mass enhancement at high temperature observed in the previous section, especially the large mass enhancement reported in table 1, is not physical but just an artifact arising from the variation of $N_{\tau}$.

In order to check this possibility, we must change $N_{\tau}$ with fixed $T$. For this purpose, we performed MEM analysis for the reconstructed correlator $G^{\mathrm{reco}}(\tau, 2 T ; T)=G(\tau)+G(\tau+1 /(2 T))$, which represents a correlator corresponding to the same spectral funciton as $G(\tau)$ but with a different $N_{\tau}$. The comparison of the spectral functions obtained with $G(\tau)$ and $G^{\text {reco }}(\tau, 2 T ; T)$ is given in figure 5 for vector channel at $T=0.78 T_{c}$. As the figure shows, the position of the $J / \psi$ peak is insensitive to the 
change of the correlator. The mass of the $J / \psi$ peak obtained with the reconstructed correlator but the same analysis as in the previous section is $\bar{m}=3.41(93)$, which agrees with the value in table 1 within the error. This result suggests that the mass enhancement of charmonia at nonzero $T$ is not an artificial one arising from the variation of $N_{\tau}$.

\section{6 summary}

In this proceedings, we studied the properties of charmonia at nonzero momentum in the vacuum and in medium with the lattice Euclidean correlation functions in the pseudoscalar and vector channels with MEM. The transverse and longitudinal components for the vector channel are analyzed separately. In addition to the standard analysis of spectral functions, we focused on the residue and dispersion relations for charmonia. To analyze these quantities with error in MEM, we have introduced the definitions in Eqs. (8).

Our results suggest that the momentum dependence of the charmonia hardly changes from the Lorentz covariant one even well above $T_{\mathrm{c}}$, although the rest mass $m$ is significantly increased. In vector channels, we do not observe difference between the transverse and longitudinal components within the error in MEM analysis even at finite temperature. These results are nontrivial because Lorentz symmetry is lost in medium, and quite interesting from the phenomenological points of view.

This work is supported in part by JSPS KAKENHI Grant Numbers 26400272 and 17K05442. Numerical simulations for this study were carried out on IBM System Blue Gene Solution at KEK under its Large-Scale Simulation Program (No. 14/15-15 and 15/16-10).

\section{References}

[1] T. Matsui, H. Satz, Phys. Lett. B 178, 416 (1986)

[2] M. Asakawa, T. Hatsuda, Phys. Rev. Lett. 92, 012001 (2004), hep-lat/0308034

[3] S. Datta, F. Karsch, P. Petreczky, I. Wetzorke, Phys. Rev. D 69, 094507 (2004), hep-lat/0312037

[4] T. Umeda, K. Nomura, H. Matsufuru, Eur. Phys. J. C 39, 9 (2004), hep-lat/0211003

[5] A. Jakovác, P. Petreczky, K. Petrov, A. Velytsky, Phys. Rev. D 75, 014506 (2007), hep-lat/0611017

[6] WHOT-QCD Collaboration, H. Ohno, S. Aoki, S. Ejiri, K. Kanaya, Y. Maezawa, H. Saito, T. Umeda, Phys. Rev. D 84, 094504 (2011), 1104 . 3384

[7] H.T. Ding, A. Francis, O. Kaczmarek, F. Karsch, H. Satz, W. Soeldner, Phys. Rev. D 86, 014509 (2012), 1204.4945

[8] S. Borsányi, S. Dürr, Z. Fodor, C. Hoelbling, S.D. Katz, S. Krieg, S. Mages, D. Nógrádi, A. Pásztor, A. Schäfer et al., J. High Energ. Phys. 2014, 1 (2014), 1401.5940

[9] M. Asakawa, Y. Nakahara, T. Hatsuda, Prog. Part. Nucl. Phys. 46, 459 (2001), hep-lat/0011040

[10] S. Datta, F. Karsch, P. Petreczky, I. Wetzorke, J. Phys. G: Nucl. Part. Phys. 31, S351 (2005), hep-lat/0412037

[11] A. Ikeda, M. Asakawa, M. Kitazawa, Phys. Rev. D95, 014504 (2017), 1610.07787

[12] M. Jarrell, J.E. Gubernatis, Phys. Rep. 269, 133 (1996)

[13] G. Aarts, C. Allton, J. Foley, S. Hands, S. Kim, Phys. Rev. Lett. 99, 022002 (2007), hep-lat/0703008

[14] C. Nonaka, M. Asakawa, M. Kitazawa, Y. Kohno, J. Phys. G: Nucl. Part. Phys. 38, 124109 (2011) 